\title{
Investigation on automated Multiple Decade Ratios precision Divider for Generation of low DC Voltages
}

\author{
Roberto Cerri, Davide Corona, Flavio Galliana ${ }^{1}$ \\ National Institute of Metrological Research, Strada Cacce, 91, 10135 Turin, Italy.
}

\begin{abstract}
At the National Institute of Metrological Research (INRIM) an automatable precision DC voltage fixed-ratios divider allowing the division ratios of DC Voltages from $10: 1$ to $10^{7}: 1$. It can be quickly calibrated when necessary and involved in traceability transfer. Its resistors are selected bulk metal foils ones connected in series in four terminal configuration whose values are $90 \mathrm{k} \Omega, 9 \mathrm{k} \Omega, 900 \Omega, 90 \Omega, 9 \Omega, 0.9 \Omega$, $90 \mathrm{~m} \Omega$ and $10 \mathrm{~m} \Omega$. The main advantage of this divider is that it can be automatically calibrated with a calibrator and a digital multimeter. Its calibration starts from a10 $\mathrm{V}$ value. It takes advantage of the DMM linearity, in particular in the $10 \mathrm{~V}$ range that allows improving its calibration uncertainty. After calibration, it can be used to divide DC Voltages lower than $10 \mathrm{~V}$. Preliminary evaluation of its calibration uncertainties and mid-term stabilities (a week) span respectively from $1.4 \times 10^{-6}$ to $6.0 \times 10^{-4}$ and from $2.4 \times 10^{-7}$ to $4.5 \times 10^{-4}$ for ratios from $10: 1$ to $10^{7}: 1$. This divider could be involved in the calibration of nanovoltmeters in a typical range from $10 \mathrm{~V}$ till down to $100 \mathrm{nV}$.
\end{abstract}

\section{Introduction}

Today the traceability from the DC Voltage standard to low and ultra-low values is still a challenge due to the needs in research, nanotechnology and medical frameworks. National and high level secondary laboratories have used for calibration of digital multi-meters (DMMs) and multifunction calibrators (MFCs) commercial high precision manually operating DC voltage dividers $[1,2]$ or a recently developed automated fixed ratios divider [3]. In addition, research on DC Voltage divider has been wide. Effective guarded dividers were developed for high accuracy DC voltage applications $[4,5]$ also for high voltages $[6-$ 10]. Problems arise for lower voltages. The widespread instrument to measure low DC Voltages is the nanovoltmeter. Modern nanovoltmeters are highly sophisticated and accurate instruments often involved in precise and highly advanced measurement applications. Unfortunately, many of them are not calibrated and used trusting in their manufacturer specifications. At high level, National Measurement Institutes (NMIs) nanovoltmeters are calibrated vs. the Josephson Voltage Standards (JVS) changing the microwave frequency $[11,12]$. Nevertheless, this choice is expensive and time consuming.

${ }^{1}$ Corresponding author: f.galliana@inrim.it. 
NMIs and Laboratories without the JVS need alternative solutions. Interesting and valid solutions with easily available instruments are proposed in [13, 14]. At National Institute of Metrological Research (INRIM) an automatable multiple decade ratios precision divider has been built which is currently being automated.

\section{The INRIM DC Voltage divider}

This divider is made with $90 \mathrm{k} \Omega, 9 \mathrm{k} \Omega, 900 \Omega, 90 \Omega, 9 \Omega, 0.9 \Omega, 90 \mathrm{~m} \Omega$ and $10 \mathrm{~m} \Omega$ resistors connected in four terminal configuration. The advantage of this divider lies in its easy and quick calibration when necessary with a calibrated DC Voltage calibrator and a particular DMM, widespread in National Measurement Institutes (NMIs) and in electrical calibration laboratories as [15]. Once the divider is calibrated it can be involved in a measurement setup, with a DC Voltage reference standard or a calibrated MFC, for the generation of low DC Voltages and calibration of nanovoltmeters mainly in the range from $100 \mathrm{nV}$ to $10 \mathrm{~V}$. Its scheme is shown in Fig. 1.

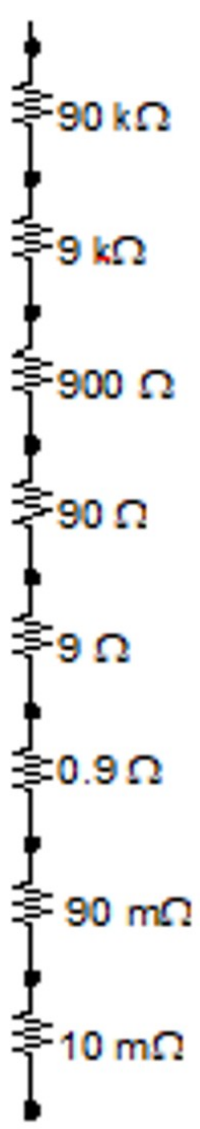

Fig. 1. Scheme of the INRIM divider for generation of DC Voltage low values.
The divider has been made with high nominal power resistors with respect to the applied power in during the divider calibration of ratios calibration. The ratio between these two powers has been kept as high as possible to neglect the effects of the power coefficients. It has been made with selected Vishay low temperature coefficient (TCR) resistors and with satisfactory short-term stability. The bistable relays have a very low contact resistance (few $\mathrm{m} \Omega$ ) being equipped with silver/gold contacts with thermo-electromotive forces (emfs) on closing contacts extremely small $\cong 40 \mathrm{nV}$. To reduce noises due to self-heating or to electrical noise due to their excitation and maintenance voltages, taking advantage of their bistable feature, their activation is made by coupling their coils in alternating regime sending them only a single pulse. Therefore, no voltage is present during the measurement on the excitation coils of the relays. The printed circuit, in vetronite, has been built with a micro milling technique for ultra-high insulation between the tracks. The coppered surfaces have double thickness than the standard. Resistors have been inserted in holes of a copper box to minimize electrical and thermal noises. Relays connections have been made very short to minimize parasitic resistances. The coils and the control circuits are supplied by a $5 \mathrm{~V} \mathrm{DC}$ Voltage avoiding the noise due to mains. The condition of the relays, that currently is carried out with switches, is constantly monitored by switching on Led diodes, activated by auxiliary contacts, allowing to detect a possible incorrect operation. Solderings have been carried out with tin and specific flux for low emfs. 


\subsection{Building details}

In Fig. 2 a photo of the developed prototype, shows its characteristics. The resistors of the resistive divider, to avoid immunity to electrical and thermal noises, have been inserted in a milled copper box. The connections of the relays to the resistors are very short to reduce the parasitic resistance of the connections by bringing them as close as possible to the copper box to improve also the thermal anchoring.

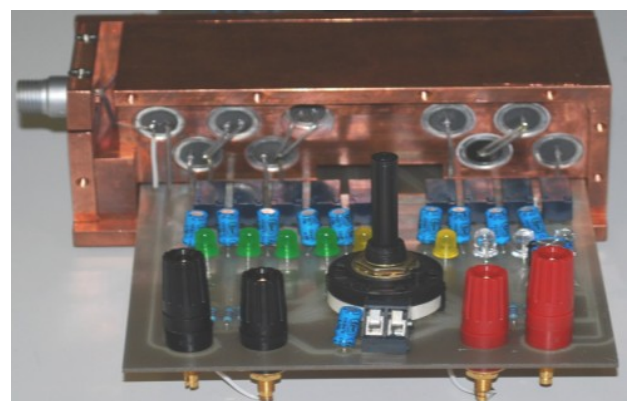

Fig. 2. Photo of the INRIM divider, in which it can be seen the copper box housing the resistors.

The resistors are placed in the box cavities which, by means of a thin layer of thermally conductive paste.

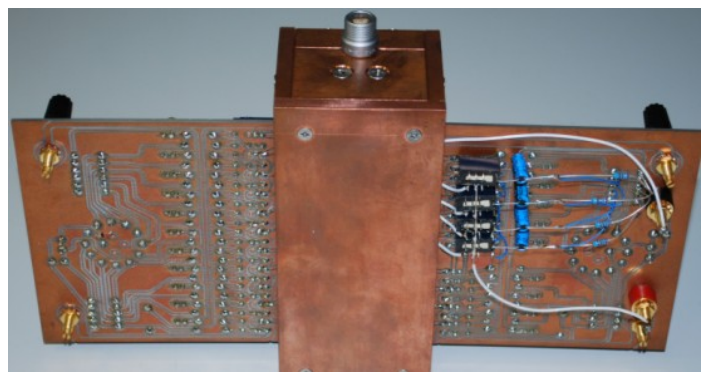

Fig. 3. Photo of the connections of the resistors of the INRIM divider, allowing their connection in four terminal configuration.

It can be noted in Figure 3 a series of additional components wired on the welding side of the printed circuit, have been added to allow a four-wire connection.

\section{Calibration of the INRIM DC Voltage divider}

It is possible to calibrate the divider when necessary updating the values its division ratios. A quick calibration can be made with the setup of Fig. 4. This setup involves the DMM [15], characterized in linearity on its $10 \mathrm{~V}$ range according to the suggestion of $[16,17]$ and a J. Fluke 5700A calibrator [18] as high stability DC voltage generator. The measurement process, for each section, consists in measuring the input voltage to a section of the divider and alternatively the output voltage ten times lower. A DC Voltage from a high stability DC Voltage calibrator is applied to a resistor, while the DMM measures this voltage. Then, leaving unchanged the supplying point and the voltage value, the DMM measures the voltage on the one-decade lower value resistor without changing the DMM range. Applying in cascade this strategy from the first section to the last one, it is possible to obtain the ratios from $10: 1$ to $10^{7}: 1$. 


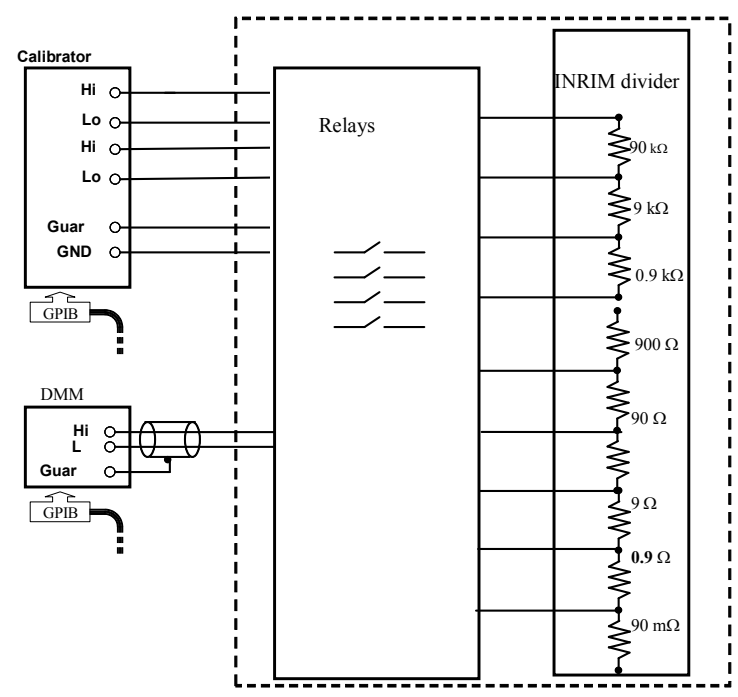

Fig. 4. Measurement setup for the quick calibration of the divider.

For example considering the section with an input resistance of $10 \mathrm{k} \Omega$, the $10 \mathrm{~V}\left(L V_{H}\right)$ voltage is applied to it and measured with the DMM on the $10 \mathrm{~V}$ range. Then, leaving unchanged the point of application of the $10 \mathrm{~V}$, the DMM will read the output on $900 \Omega$ corresponding (taking into account the resistors from $900 \Omega$ to ground) at $1 \mathrm{k} \Omega$ (1 V always on the $10 \mathrm{~V}$ range). The ratio between the input on $10 \mathrm{k} \Omega$ and the output on $900 \Omega$ will therefore be evaluable. The same procedure is repeated at $-10 \mathrm{~V}$ to lower the emfs effects. The procedure can include, before the polarities reversal and before each section change, the evaluation of the zeroes to be eventually added to the following DMM readings. The unknown ratio values are given meaning the values at both polarities from the following relation:

$$
\mathrm{R}=\frac{\mathrm{L}_{\mathrm{H}}}{\mathrm{L}_{\mathrm{L}}}
$$

Where $\mathrm{R} \cong 10$ is the ratio, $L_{H}$ is the supplying voltage while $L_{L}$ is the voltage on the lower decade resistor. Those voltages are the mean voltage readings by the DMM. In Table 1, the involved resistors, voltages and DMM readings for each divider section, are listed.

Table 1. Resistors, voltages and DMM ranges for the quick calibration of the INRIM divider.

\begin{tabular}{|c|c|c|c|c|c|c|}
\hline Section & $\begin{array}{c}\text { Cumulative } \\
\text { ratio }\end{array}$ & $\begin{array}{c}\text { Resistor to apply } \\
\mathrm{V}_{\mathrm{H}}\end{array}$ & $\begin{array}{c}\text { Resistor to } \\
\text { measure } \mathrm{L}_{\mathrm{L}}\end{array}$ & $\begin{array}{c}\mathrm{L}_{\mathrm{H}} \\
\text { (V) }\end{array}$ & $\begin{array}{c}\mathrm{L}_{\mathrm{L}} \\
\text { (V) }\end{array}$ & $\begin{array}{c}\text { DMM } \\
\text { range }\end{array}$ \\
\hline 1 & $10: 1$ & $90 \mathrm{k} \Omega$ & $10 \mathrm{k} \Omega$ & 10 & 1 & 10 \\
2 & $10^{2}: 1$ & $9 \mathrm{k} \Omega$ & $1 \mathrm{k} \Omega$ & 10 & 1 & 10 \\
3 & $10^{3}: 1$ & $900 \Omega$ & $100 \Omega$ & 1 & 0.1 & 1 \\
4 & $10^{4}: 1$ & $90 \Omega$ & $1 \Omega$ & 1 & 0.1 & 1 \\
5 & $10^{5}: 1$ & $9 \Omega$ & $1 \Omega$ & 0.1 & $10^{-2}$ & 0.1 \\
6 & $10^{6}: 1$ & $9 \Omega^{2}$ & $90 \mathrm{~m} \Omega$ & $10^{-2}$ & $10^{-3}$ & 0.1 \\
7 & $10^{7}: 1$ & $9 \Omega^{3}$ & $10 \mathrm{~m} \Omega$ & $10^{-3}$ & $10^{-4}$ & 0.1 \\
\hline
\end{tabular}

\footnotetext{
${ }^{2} V_{H}$ is applied on the $9 \Omega$ resistor to avoid excessive load on the calibrator, but $L_{H}$ is measured on the $900 \mathrm{~m} \Omega$ resistor.

${ }^{3} V_{H}$ is applied on the $9 \Omega$ resistor to avoid excessive load on the calibrator, but $L_{H}$ is measured on the $90 \mathrm{~m} \Omega$ resistor.
} 
To take into account all the input quantities in the uncertainty budget for the quick calibration of the divider, the (1) can be re-written as:

$$
\mathrm{R}=\frac{\mathrm{L}_{\mathrm{H}} \varepsilon_{\text {linH }} \varepsilon_{\mathrm{G}} \varepsilon_{\mathrm{emfH}}}{\mathrm{L}_{\mathrm{L}} \varepsilon_{\text {linL }} \varepsilon_{\mathrm{emfl}}} \cdot \varepsilon_{\text {load }}
$$

- $\varepsilon_{\operatorname{linH}}$ and $\varepsilon_{\operatorname{linL}}$ are the DMM linearity specifications at $10 \mathrm{~V}$ and $1 \mathrm{~V}$;

- $\varepsilon_{\text {iemfo }}$ and $\varepsilon_{\text {lemfL }}$ are the are the emfs when the DMM measures $10 \mathrm{~V}$ and $1 \mathrm{~V}$;

$\varepsilon_{\mathrm{G}} \varepsilon_{V c}$ and $\varepsilon_{\text {load }}$ are respectively the corrections due to the stability of calibrator to the voltage coefficient of the divider and to the load effect at the DMM input.

\subsection{Uncertainties of the quick calibration method of the divider}

Let's consider the example of the previous paragraph where on an input resistance of $10 \mathrm{k} \Omega$ is supplied a DC Voltage of $10 \mathrm{~V}$. Taking advantage of the DMM linearity specifications on the $10 \mathrm{~V}$ range, it is possible consider these ones instead of the much larger accuracy specifications. In Table 2, the standard uncertainties budgets of the quick calibration of this section is given.

Table 2. Standard uncertainties of the quick calibration of the first section of the INRIM divider.

\begin{tabular}{|l|c|c|}
\hline \multicolumn{1}{|c|}{$\begin{array}{c}\text { Uncertainty } \\
\text { component }\end{array}$} & type & $1 \delta\left(\times 10^{-7}\right)$ \\
\hline DMM noise of $\mathrm{L}_{\mathrm{H}}$ & $\mathrm{A}$ & 0.3 \\
$\mathrm{DMM}$ noise of $\mathrm{L}_{\mathrm{L}}{ }$ & $\mathrm{A}$ & 0.1 \\
$\varepsilon_{\mathrm{linH}}$ & $\mathrm{B}$ & 0.6 \\
$\varepsilon_{\mathrm{linL}}$ & $\mathrm{B}$ & 3.2 \\
$\varepsilon_{\mathrm{G}}$ & $\mathrm{B}$ & 2.3 \\
$\varepsilon_{\mathrm{eVc}}$ & $\mathrm{B}$ & 0.0 \\
$\varepsilon_{\mathrm{emfH}}$ & $\mathrm{B}$ & 0.1 \\
$\varepsilon_{\mathrm{emfL}}$ & $\mathrm{B}$ & 1.2 \\
$\varepsilon_{\text {load }}$ & $\mathrm{B}$ & $5.8^{5}$ \\
\hline RSS & & 7.1 \\
\hline
\end{tabular}

In these first characterization measurements, the divider has been used in a semiautomated way, selecting the desired ratios d ratios by means of a rotary selector. In table 3, are reported the standard uncertainties of each section and the standard uncertainty of the cumulative ratios. These ratios are obtained multiplying the 10:1 ratios of each section.

\footnotetext{
${ }^{4}$ This measurement is also made on the $10 \mathrm{~V}$ range of the DMM.

${ }^{5}$ This component has been evaluated considering the input impedance of a specific DMM. The input impedance of the available DMMs model [15] were accurately measured and the item with the higher impedance (about $8.6 \times 10^{11} \Omega$ ) was enrolled in the measurement setup.
} 
Table 3. Uncertainties of the quick calibration of the each section and of the cumulative ratios of the INRIM divider.

\begin{tabular}{|c|c|c|c|c|}
\hline Section & $u\left(\times 10^{-6}\right)$ & $\begin{array}{c}\text { Cumulative } \\
\text { Ratios }\end{array}$ & $u\left(\times 10^{-6}\right)$ & $U\left(\times 10^{-6}\right)$ \\
\hline 1 & 0.7 & $10: 1$ & 0.7 & 1.4 \\
2 & 0.4 & $10^{2}: 1$ & 0.9 & 1.7 \\
3 & 1.5 & $10^{3}: 1$ & 1.7 & 3.4 \\
4 & 1.5 & $10^{4}: 1$ & 2.3 & 4.6 \\
5 & 6.8 & $10^{5}: 1$ & 7.0 & 14 \\
6 & 30.5 & $10^{6}: 1$ & 32.5 & 63 \\
7 & 296 & $10^{7}: 1$ & 296 & 592 \\
\hline
\end{tabular}

The uncertainties of the cumulative ratios are evaluated taking into account a partial correlation $\left(r=1.6 \times 10^{-4}\right)$ in the evaluation of the cumulative ratios. $r$ was evaluated according to [19].

\section{Discussion}

In addition to the described work, an analysis of the short-time stability of the divider ratios has been made. In fact, as it can be quickly calibrated when necessary and involved in traceability transfer, as its involvement in a measurement setup for calibration of nanovoltmeters. Table 4 shows the preliminary values of the short-time (a week) stability of the divider ratios.

Table 4. Short-time stability of the divider ratios.

\begin{tabular}{|c|c|}
\hline Ratios & Stability $\left(\times 10^{-6}\right)$ \\
\hline $10: 1$ & 0.3 \\
$10^{2}: 1$ & 0.3 \\
$10^{3}: 1$ & 0.7 \\
$10^{4}: 1$ & 0.8 \\
$10^{5}: 1$ & 6.6 \\
$10^{6}: 1$ & 47.6 \\
$10^{7}: 1$ & 446 \\
\hline
\end{tabular}

These values have to be confirmed in successive evaluations of the divider and inserted in the divider use uncertainties [20], along with its calibration uncertainties. Use uncertainties are those with which it can be used when involved in calibration of other instrumentation though the divider is realized to be quickly calibrated when necessary to be used shortly after. In Fig. 5, the mid-term stability of the 10:1 ratio is shown. 


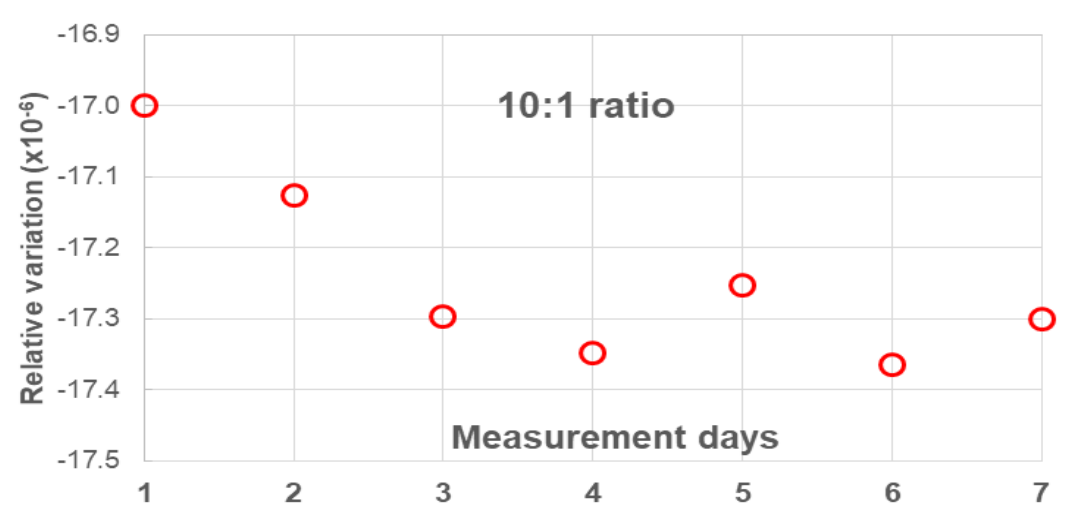

Fig. 5. Mid-term stability of the 10:1 ratio.

\section{Conclusion}

From preliminary results, the performance of the divider are satisfactory. Next work will be the full automation of the divider and the further verification of its measurement noises in the calibration and of its short-term stabilities. Consequently, the calibration uncertainties could be re-evaluate as well as its short-time use uncertainties can be evaluated.

\section{References}

1. Fluke Corporation, 752 A reference divider Instruction Manual, Rev1, (1984).

2. Fluke Corporation, 720 A Kelvin Varley voltage divider instruction Manual, Rev. 2, (1974).

3. F.Galliana, P.P. Capra R. Cerri, M. Lanzillotti, Measurement 122, pp. 291-296, (2018).

4. Y. Sakamoto, T. Endo, H. Shao, S. Matsuzawa, Prec. El. Measur. Conf. CPEM, pp. 363-364, (2000).

5. Tadashi Endo, Y. Sakamoto, T. Sakuraba, Y. Murayama, H. Yoshida, A. Odawara, IEEE Trans. Instrum. Meas. Vol 40 (2), pp. 333- 336, (1991).

6. H.Hirayama ; M. Kobayashi, K. Murakami, T. Kato, IEEE Trans. Instrum. Meas. 23 (4) pp. 314- 317, (1974).

7. Yi Li, Miyuru K. Ediriweera, F. S. Emms, A. Lohrasby, IEEE Trans. Instrum. Meas. 60 (7), pp. 2211-2216 (2001).

8. R.B.D. Knight; P. Martin, IEEE Trans. Instrum. Meas. 54 (5), pp. 568-570, (2005).

9. N. Dragounova, IEEE Trans. Instrum. Meas. 42 (2), pp. 1911-1915, (1993).

10. A.Mereva, Ö. Kalenderli, Journal of Electrostatics 67 (5) pp. 741-745, (2009)

11. D. Georgakopoulos, S. Grady, I. Budovsky, S. Grady,IEEE Trans. Instrum. Meas. 42 (2), pp. 1581-1585, (2013).

12. H. E van den Brom, E.t Houtzager, G. Rietveld, R.van Bemmelen and O. Chevtchenko Meas. Sci.Tech, 18, (11), (2007).

13. D. E Murray, C. Laurie, T. B. Lawson, 29th. Prec. El. Measur. Conf. CPEM, pp. 138139, (2014).

14. D. Aviles, E. Navarrete, 31th. Prec. El. Measur. Conf. CPEM, pp. 138-139, DOI: 10.1109/CPEM.2018.8501104, (2018).

15. Agilent Technologies, 3458A multimeter - User's Guide, rev. B.01, (2001). 
16. A. Sosso, R. Cerri, Prec. El. Measur. Conf. CPEM, pp. 375-376, (2000).

17. J. I. Giem, IEEE Trans. Instrum. Meas., 40 (2), 329-332, (1991).

18. Fluke Corporation, 5700A/5720A Series II Multi-function calibrator service manual, Rev. 1, 3/02, (June 1996).

19. F.Galliana, M. Lanzillotti, Measurement 102, pp. 353-360, (2017).

20. W. Bich, F. Pennecchi, Advanced Mathematical \& Computational Tools in Metrology, World Scientific, pp. 59-169, (2004). 